\title{
Atomic-velocity class selection using quantum interference
}

\author{
D. Wilkowski, J. C. Garreau, D. Hennequin, and V. Zehnlé \\ Laboratoire de Spectroscopie Hertzienne, associé au CNRS, Centre d'Etudes et de Recherches Lasers et Applications, \\ Université des Sciences et Technologies de Lille, F-59655 Villeneuve d'Ascq Cedex, France
}

(Received 27 October 1995; revised manuscript received 11 March 1996)

\begin{abstract}
A method enabling atomic velocity class selection by means of quantum interference in the two-photon ionization of an atom through two quasiresonant intermediate levels is studied. This method is compatible with the Doppler cooling in optical molasses, and it is able to attain temperatures colder than the Doppler limit. The advantages and limitations of this method are discussed. We study the effect of the competition with the Doppler cooling for temperatures lower than the Doppler limit, when the usual Doppler process heats the atoms rather than cools them. The method is shown to be limited essentially by the loss of ground-state atoms due to ionization. We also propose and study a "source" scheme in which new atoms are continuously injected into the system, leading to a nonvanishing stationary number of cold atoms. Finally, we propose generalizations of the method that allows us to combine it with Sisyphus-type mechanisms. [S1050-2947(96)00610-5]
\end{abstract}

PACS number(s): 32.80.Pj, 42.50.Vk, 32.80.Rm

\section{INTRODUCTION}

In the last ten years, laser cooling of atoms has become one of the major and most promising subjects in atomic physics and quantum optics, and a great deal of both theoretical and experimental effort has been done in this field. The theoretical limits for the minimum temperature have been progressively overcome. The so-called Doppler limit (typically a hundred of microkelvins) has been beaten by Sisyphus-type mechanisms [1] and the minimum temperature pushed down to the photon recoil limit (or spontaneous emission limit), of the order of a few microkelvins. Two methods relying on laser-atom interactions have been experimentally demonstrated to allow cooling below the photon recoil limit: velocity selection by coherent population trapping (VSCPT) [2] and Raman subrecoil cooling [3]. A third method, the so-called "evaporative cooling" [4-7] that is not based on laser-atom interactions, and thus not limited by spontaneous emission effects, has led to record low temperatures of a few nanokelvins and has recently allowed experimental observations of degenerate-gas Bose-Einstein condensation [5-7].

In a recent paper [8], the possibility of using quantum interference in the two-photon ionization of an atom through two quasiresonant intermediate levels in order to generate quantum coherence has been suggested. In particular, it has been shown that quantum coherence can be generated between internal and external degrees of freedom of the atom, thanks to the Doppler effect, allowing selection of a particular velocity class in a velocity distribution profile.

In the present paper we shall study the performances of this velocity selection method. We show that the redistribution of atoms among the velocity classes due to the spontaneous emission from the intermediate levels does not limit the lowest temperature obtainable, provided that a sufficiently high ionization rate can be achieved. There is, nevertheless, another limitation: as the method is based on the irreversible ionization of the atoms, the ions eventually escape the volume of interaction with the laser and are lost.
The lower the temperature, the smaller the number of remaining, cold, ground-state atoms. The present method is compatible with the usual laser cooling schemes, so that this depopulation effect can be reduced by first cooling the atoms by the usual methods. However, if the temperature becomes lower than the Doppler limit, the Doppler mechanism tends to heat the atoms. We shall study how the competition of these two effects influences the performances of the quantum interference velocity selection.

\section{VELOCITY SELECTION BY QUANTUM INTERFERENCE}

In this section we recall the basic mechanism of velocity class selection using quantum interference in the two-photon atomic ionization. For a discussion of this process as a mean of generating quantum coherence see Ref. [8]. We shall restrict ourselves here to the one-dimensional case.

Consider the atomic system shown in Fig. 1. The ground state is coupled to the continuum by two modes of the electromagnetic field (characterized by photon numbers $n_{1}$ and $n_{2}$ and wave numbers $k_{1}$ and $k_{2}$ ) through two quasiresonant intermediate levels, having the same natural width $\Gamma$. Mode 1 (2) couples the ground state to the intermediate level $\left|e_{1}\right\rangle$ $\left(\left|e_{2}\right\rangle\right)$, with a dipole matrix element $v_{1}\left(v_{2}\right)$ and the intermediate level $\left|e_{2}\right\rangle\left(\left|e_{1}\right\rangle\right)$ to the continuum, with a dipole matrix element $v_{2}^{\prime}\left(v_{1}^{\prime}\right)$ and laser-atom detuning $\delta_{1}\left(\delta_{2}\right)$. We take the two modes to be counterpropagating $\left(k_{2}=-k_{1}\right.$ $=k=\omega / c)$ and the atomic center-of-mass velocity to be $v$. In order to simplify formulas, we will also take $v_{1} v_{1}^{\prime}$ $=-v_{2} v_{2}^{\prime}$, but this hypothesis is not at all essential: the same interference effect can be obtained by adjusting the detunings. We neglect noninterfering terms corresponding to the ionization due to the absorption of two photons from the same mode [8] (see also Sec. VI).

As a consequence of these couplings, the atomic ground state acquires a finite lifetime corresponding to an ionization rate given by [8] 


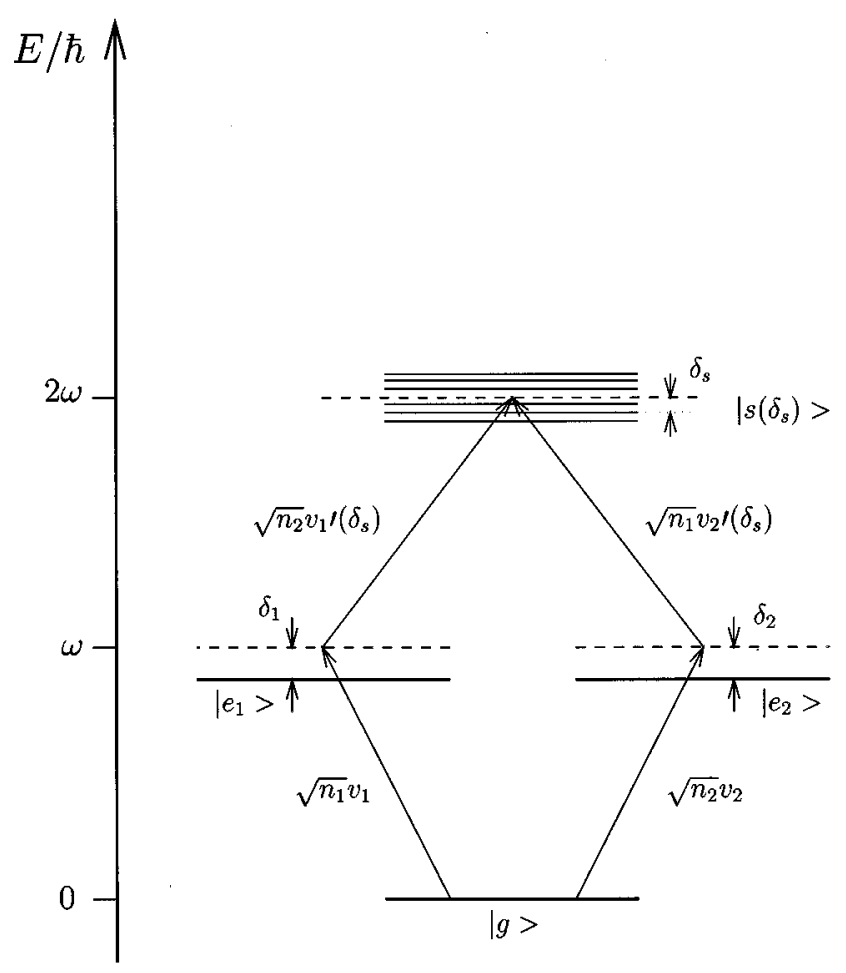

FIG. 1. Level structure and electromagnetic couplings for the "atom."

$$
\Gamma_{g}=\Gamma_{0} \omega^{2} \frac{\left(\delta_{2}-\delta_{1}-2 k v\right)^{2}}{\left[\left(\delta_{1}+k v\right)^{2}+\Gamma^{2} / 4\right]\left[\left(\delta_{2}-k v\right)^{2}+\Gamma^{2} / 4\right]},
$$

where we took into account the Doppler shifts $( \pm k v)$ of the atomic transitions, and defined the constant $\Gamma_{0}=\left(2 \pi / \hbar^{2}\right) f_{0} \omega^{2} n_{1} n_{2}\left|v_{1} /(\hbar \omega)\right|^{2}\left|v_{1}^{\prime} /(\hbar \omega)\right|^{2}$, where $f_{0}$ is the density of states of the continuum. We also regularized the denominators by adding the term $\Gamma^{2} / 4$, which corresponds to add a negative imaginary part $-i \hbar \Gamma / 2$ to the energy of the intermediate levels in order to take into account their finite lifetime $\Gamma^{-1}$. This procedure is usual in scattering theory and corresponds to a renormalization of the evolution operator by taking into account its coupling to the vacuum modes (which is responsible for spontaneous emission).

Equation (2.1) shows that the ionization rate can vanish (corresponding to an infinite lifetime for the ground state) if the condition

$$
\delta_{2}-\delta_{1}=2 k v
$$

is satisfied. The vanishing of the transition rate is due to quantum interference between the two paths connecting the ground state to the continuum via the intermediate states [8]. This interference is at the heart of the velocity selection method studied in this paper. The atoms in the velocity class $v=\left(\delta_{2}-\delta_{1}\right) / 2 k$ will stay in the ground state, whereas all other atoms will be eventually ionized. Obviously, the velocity selection is obtained at the price of a decrease of the number of atoms in the ground state. The creation of a coherence between the ground state and a particular velocity class allows us, thus-by subsequent filtering of the groundstate atoms - to select this particular velocity class.

The ionization being an irreversible process (we neglect the usually very small recombination probability), the velocity selection mechanism proposed here is in principle not limited by spontaneous emission, and is thus able to cool the system to a temperature below the photon-recoil limit (see Sec. VI).

In order to have an insight into the velocity selection process, we consider a simple concrete example: let us take equal detunings $\left(\delta_{1}=\delta_{2}=\delta\right)$ and suppose that $|\delta| \gg \Gamma$, so that the population of the intermediate levels is very small, and the effect of redistribution of atoms among the velocity classes by the spontaneous emission can be neglected. The system will thus select the $v=0$ velocity class. Suppose that the atoms have initially a Maxwellian distribution

$$
n_{0}(v)=\frac{N_{0}}{v_{0} \sqrt{2 \pi}} \exp \left[-\left(v / \sqrt{2} v_{0}\right)^{2}\right]
$$

corresponding to an initial temperature $T_{0}=M v_{0}^{2} / k_{B}\left(k_{B}\right.$ is Boltzmann's constant and $M$ the mass of the atom). If the initial velocity dispersion $v_{0}$ is small enough $\left(k v_{0} \ll \delta\right)$, the transition rate given by Eq. (2.1) can be expanded to the lowest order in $v$, namely, $v^{2}: \Gamma_{g}=G\left(v / v_{0}\right)^{2}+O\left(v^{4}\right)$. The time evolution of the velocity distribution for the remaining ground-state atoms is given by

$$
n(v, t)=\frac{N_{0}}{v_{0} \sqrt{2 \pi}} \exp \left[-(1+2 G t)\left(v / \sqrt{2} v_{0}\right)^{2}\right]
$$

showing that the distribution keeps a Gaussian shape. The kinetic temperature $T(t)$ of the system is thus reduced by the factor $\left(T / T_{0}\right)=1 /(1+2 G t)$. The number of remaining atoms at time $t$ is also easily computed

$$
N(t)=\int n(v, t) d v=\frac{N_{0}}{\sqrt{1+2 G t}},
$$

from which one deduces that the relative decrease in the temperature is proportional to the square of the relative decrease of the number of atoms: $\left[T(t) / T_{0}\right]=\left[N(t) / N_{0}\right]^{2}$.

These results show that in the case considered here, where the redistribution of atoms among the atomic velocity classes by spontaneous emission is absent, there is no lower limit for the temperature, and that the process is limited only by the decrease in the number of remaining ground-state atoms. In practice, however, one should work closely to resonance in order to have high ionization probabilities, and the process of redistribution of velocities by spontaneous emission is no longer negligible. The influence of this process in the velocity selection method described above will be studied in the next section.

\section{EFFECT OF THE SPONTANEOUS EMISSION FROM THE INTERMEDIATE LEVELS}

The level scheme shown in Fig. 1 is, if we neglect the coupling to the continuum, a usual one for Doppler cooling in optical molasses, provided the detunings are negative. The 
atoms are cooled by preferential absorption of photons in the laser wave counterpropagating with respect to their motion [9]. This absorption is followed by spontaneous emission in a random direction, so that after many fluorescence cycles there is a net reduction of the velocity until the system attains an equilibrium temperature called "Doppler limit temperature' $T_{D}$. It is clear that if the selective ionization discussed in the preceding section continues to cool the system below this limit, the existence of the fluorescence cycles involving the intermediate states and the ground state will tend to heat the system. In other words, for temperatures below the Doppler limit there will be competition between the two processes.

We shall deal with the effect of spontaneous emission using a Fokker-Planck type approach. For a rigorous derivation of the Fokker-Planck equation for the optical molasses and a discussion of the limits of this approach, see Refs. [10-12]. We consider here what we shall call an "ionization-assisted" optical molasses (IAOM), with no trapping system, so that we do not have to take into account the atomic positions. We also consider that, once ionized, the atom escapes the molasses and is lost, thus effectively performing the velocity selection. The two laser waves have the same intensity $\left(n_{1}=n_{2}\right)$, and the dipole matrix elements are equal for the two intermediate states $\left(\left|v_{1}\right|=\left|v_{2}\right|\right.$, $\left.\left|v_{1}^{\prime}\right|=\left|v_{2}^{\prime}\right|\right)$, which is the case if the intermediate levels are magnetic sublevels of the same atomic level. It is useful to define the resonant Rabi frequency corresponding to the transitions connecting the ground state to the intermediate states

$$
\hbar \Omega=\sqrt{n_{1}}\left|v_{1}\right|=\sqrt{n_{2}}\left|v_{2}\right|
$$

For low laser power, the transition rate from the ground state to the intermediate state $\left|e_{i}\right\rangle$ is given by

$$
\Gamma_{i}=\frac{\Gamma}{2} s_{i}(v) \quad(i=1,2),
$$

where $s_{i}(v)$ is the so-called "saturation parameter":

$$
s_{i}(v)=\frac{\Omega^{2} / 2}{(\delta \pm k v)^{2}+(\Gamma / 2)^{2}},
$$

where the $+(-)$ sign on the denominator corresponds to mode 1 (2).

Let us denote the velocity distribution for ground-state atoms at time $t$ by $n(v, t)$ and the velocity distribution for excited atoms by $n^{*}(v, t)=n_{1}^{*}(v, t)+n_{2}^{*}(v, t)$. It turns out that only the total number of excited atoms $n^{*}$ is relevant, due to the fact that the intermediate states are coupled only through the unique ground state. This would not be the case if the ground state was degenerate.

The velocity shift due to the absorption or the emission of a photon is $\Delta=\hbar k / M$. The rate equation for the velocity distribution of the intermediate-state atoms is thus (we drop in the following the parameter $t$ in the arguments of $n$ and $\left.n^{*}\right)$

$$
\begin{aligned}
\frac{\partial n^{*}(v)}{\partial t}= & \dot{n}^{*}(v)=\frac{\Gamma}{2} s_{1}(v+\Delta) n(v+\Delta) \\
& +\frac{\Gamma}{2} s_{2}(v-\Delta) n(v-\Delta)-\Gamma n^{*}(v)
\end{aligned}
$$

The first two terms on the right-hand side correspond to the atoms arriving at the intermediate states $\left|e_{1}\right\rangle$ and $\left|e_{2}\right\rangle$, respectively, whereas the last term describes the depopulation of these levels by spontaneous emission.

A corresponding rate equation can be written for groundstate atoms

$$
\begin{aligned}
\dot{n}(v)= & -\frac{\Gamma}{2} s_{1}(v) n(v)-\frac{\Gamma}{2} s_{2}(v) n(v)-\Gamma_{g}(v) n(v) \\
& +\frac{\Gamma}{2} n^{*}(v+\Delta)+\frac{\Gamma}{2} n^{*}(v-\Delta) .
\end{aligned}
$$

The first two terms on the right-hand side correspond to the transitions to the intermediate states, the third term represents the depopulation due to the two-photon ionization and the last two terms are due to the repopulation of the ground state by spontaneous emission from the intermediate states.

For the weak couplings encountered in laser cooling, the population of the intermediate states can be adiabatically eliminated, giving an expression for $n^{*}(v)$ that is easily obtained by putting $\dot{n}^{*}=0$ in Eq. (3.4). The substitution of the resulting expression in Eq. (3.5) produces the following rate equation for the ground-state velocity distribution:

$$
\begin{aligned}
\dot{n}(v)= & -\frac{\Gamma}{4}\left[\left[s_{1}(v)+s_{2}(v)\right] n(v)+\frac{4 \Gamma_{g}(v)}{\Gamma} n(v)\right. \\
& \left.-s_{1}(v-2 \Delta) n(v-2 \Delta)+s_{2}(v+2 \Delta) n(v+2 \Delta)\right] .
\end{aligned}
$$

With respect to the velocity, this is a finite difference equation connecting velocity classes $2 \Delta$ apart. In order to transform this rather awkward equation in a partial differential equation, we follow the standard procedure used to obtain a Fokker-Planck equation [13], based on the assumption that the velocity shift $\Delta$ is small compared to the width of the velocity profile ("limit of small jumps"). This is actually the case if the kinetic temperature satisfies $T \gg M \Delta^{2} / k_{B}$ (i.e., well above the photon recoil limit). One can then expand the expressions for $s_{i}(v \pm 2 \Delta)$ and $n(v \pm 2 \Delta)$ up to the order $\Delta^{2}$, leading to the following equation:

$$
\frac{\partial n}{\partial t}=\frac{\alpha}{M} \frac{\partial}{\partial v}(v n)+\frac{D}{M^{2}} \frac{\partial^{2} n}{\partial v^{2}}-\Gamma_{g} n,
$$

where $\alpha$ is the momentum damping coefficient given by

$$
\alpha=-2 \hbar k^{2} \frac{\delta \Gamma \Omega^{2} / 2}{\left[\delta^{2}+(\Gamma / 2)^{2}\right]^{2}}
$$

and $D$ is the momentum diffusion coefficient 


$$
D=\hbar^{2} k^{2} \frac{\Gamma \Omega^{2} / 2}{\delta^{2}+(\Gamma / 2)^{2}} .
$$

Equation (3.7) is not a Fokker-Planck equation, due to the presence of the ionization term (it does not conserve the total number of particles). If we drop the ionization term, the resulting equation is the Fokker-Planck equation describing the behavior of the Doppler optical molasses. The corresponding equilibrium temperature is given by $k_{B} T_{D}=D / \alpha$

$$
k_{B} T_{D}=-\frac{\hbar \Gamma}{4}\left(\frac{\delta}{\Gamma / 2}+\frac{\Gamma / 2}{\delta}\right)
$$

from which one can deduce the well-known result that the minimum temperature condition for the optical molasses is $\delta=-\Gamma / 2$ corresponding to the Doppler limit temperature $k_{B} T_{D}=\hbar \Gamma / 2$.

Let us now consider the complete equation with the ionization term. The problem in which we are interested here is that of the competition between the velocity selection by ionization vs Doppler cooling for temperatures of the order of, or smaller than, the Doppler limit. We will thus suppose that the system has previously been cooled to the Doppler limit temperature; we begin with a Maxwellian velocity profile of the form (2.3), with a temperature corresponding to the Doppler limit: $T_{0}=T_{D}$, or $v_{0}=v_{D}=\sqrt{k_{B} T_{D} / M}$ $=\sqrt{D / M \alpha}$. It is then useful to rewrite Eq. (3.7) in terms of the dimensionless scaled variables

$$
V=v / v_{D}
$$

for the velocity, and

$$
\tau=\alpha t / M=t / \tau_{v}
$$

for the time. $\tau_{v}=M / \alpha$ can be interpreted as the time constant characterizing the velocity damping under the action of the Doppler cooling alone. As the initial velocity dispersion is supposed small, we consider only the first term in the expansion of $\Gamma_{g}$ in powers of $V$, i.e., the term of order $V^{2}$

$$
\Gamma_{g}=(\alpha / M) g V^{2}+O\left(V^{4}\right),
$$

where the coefficient $(\alpha / M)$ has been introduced for later convenience. The dimensionless constant $g$ is given by

$g=\frac{\Gamma_{0}}{2 \Gamma}\left(\frac{\omega}{\delta}\right)^{2} \frac{1+(2 \delta / \Gamma)^{2}}{(\Omega / \Gamma)^{2}}=\pi f_{0} \Gamma \omega^{2}\left|\frac{v_{1}^{\prime}}{v_{1}}\right|^{2}\left[1+(2 \delta / \Gamma)^{2}\right] \frac{\Omega^{2}}{\delta^{2}}$.

The physical meaning of $g$ becomes clear if one writes the relation between $\Gamma_{g}$ and $g$ in the following equivalent form:

$$
\Gamma_{g}=g\left(\frac{v}{v_{D}}\right)^{2} \frac{1}{\tau_{v}}+O\left(v^{4}\right)
$$

showing that $g$ is a proportionality factor relating the ionization rate to the inverse of the characteristic time constant $\tau_{v}=M / \alpha$ through a particle-dependent factor equal to the ratio of the particle's kinetic energy to the equilibrium Doppler thermal energy.

In these scaled units Eq. (3.7) takes a simpler form [14]

$$
\frac{\partial n}{\partial \tau}=\frac{\partial(V n)}{\partial V}+\frac{\partial^{2} n}{\partial V^{2}}-g V^{2} n
$$

This equation has an analytical solution. It preserves the Maxwellian form, thanks to the approximation (3.13), but with a time-dependent width, describing the velocity selection effect. Let us write the solution in the form

$$
n(V, \tau)=f(\tau) \exp \left[-\beta(\tau) V^{2}\right] .
$$

Substituting this ansatz back in Eq. (3.16) we find, after some algebra, the expressions for $f(\tau)$ and $\beta(\tau)$. The temperature is related to the function $\beta(\tau)$ : $T(\tau) / T_{D}=[2 \beta(\tau)]^{-1}$ (the factor $T_{D}$ is a consequence of the scalings introduced above). One finds

$$
T(\tau)=T_{D} \frac{2}{\lambda \tanh (\lambda \tau+\phi)+1},
$$

where we introduced the notations

$$
\begin{gathered}
\lambda=\sqrt{4 g+1}, \\
\phi=\tanh ^{-1}\left(\frac{2 T_{D}-T_{0}}{\lambda T_{0}}\right),
\end{gathered}
$$

and $T_{0}=T(0)$. The temperature thus tends asymptotically to a stationary value, in contrast with the result obtained in the absence of spontaneous emission (see Sec. II). This stationary value is given by

$$
\frac{T_{s t}}{T_{D}}=\frac{2}{\lambda+1}
$$

and goes to zero as $\lambda^{-1} \approx g^{-1 / 2}$ for large $g$. The minimum temperature is thus not limited by the spontaneous emission from the intermediate levels, provided that the ionization rate is high enough. Note, however, that the above expression for the temperature [Eq. (3.18)] is valid only if $T_{0}>T_{s t}$. Asymptotically $(\tau \rightarrow \infty)$, the temperature approaches the stationary value as

$$
T(\tau) \approx T_{s t}+\frac{4 \lambda T_{D}}{(\lambda+1)^{2}} e^{-2 \lambda \tau} .
$$

The time constant for the relaxation of the temperature towards its stationary value is $2 \lambda$.

The solution for the function $f(\tau)$ is

$$
f(\tau)=\frac{N_{0}}{\sqrt{\pi}} \sqrt{\frac{T_{D}}{T_{0}}} \cosh \phi e^{\tau / 2} \operatorname{sech}(\lambda \tau+\phi),
$$

where $N_{0}$ is the initial number of atoms. The total number of ground-state atoms at time $\tau$ is given by the integral over $V$ of the velocity distribution and reads

$$
N(\tau)=N_{0} \cosh \phi \sqrt{1+\lambda \tanh \phi} \frac{e^{\tau / 2} \operatorname{sech}(\lambda \tau+\phi)}{\sqrt{1+\lambda \tanh (\lambda \tau+\phi)}}
$$

Asymptotically, the number of ground-state atoms goes to zero as 


$$
N(\tau) \approx N_{0}\left(1+e^{-2 \phi}\right)\left(\frac{1+\lambda \tanh \phi}{1+\lambda}\right)^{1 / 2} \exp \left[-\left(\lambda-\frac{1}{2}\right) \tau\right]
$$

showing that the time constant for the decreasing of the number of atoms is $\lambda-1 / 2$. For large ionization rates $(g \gg 1$ or, equivalently, $\lambda \gg 1$ ), it can be seen from the asymptotic expressions above that $\left(T-T_{s t}\right) / T_{D} \approx\left[N / N_{0}\right]^{2}$, in analogy with the result found in the case where there is no velocity redistribution by spontaneous emission (Sec. II).

Concluding this section, we can resume our main results: a Fokker-Planck-like equation has been written and solved describing the behavior of an ionization-assisted optical molasses in the limit of weak laser powers and low velocities. The solution shows that due to the velocity redistribution by spontaneous emission the temperature tends to an asymptotic value that is roughly proportional to the inverse of the square root of the ionization strength. In the next section we will compare the results of the above theory to Monte Carlo simulations of the behavior of an IAOM.

\section{COMPARISON OF THE THEORY WITH MONTE CARLO SIMULATIONS}

In order to verify the theory developed in the preceding section, in particular, the approximations made, we performed Monte Carlo simulations [15] for the IAOM. We begin with a Maxwellian distribution of atoms at $T_{0}=T_{D}$. For each atom in the ground state moving with a given velocity $v(0)$ inside the distribution profile, we calculate the transition rates $\Gamma_{1}$ and $\Gamma_{2}$ for absorbing a photon in modes 1 and 2 [given by Eq. (3.2)] and the rate $\Gamma_{g}$ for the two-photon ionization [given by Eq. (2.1)]. The probability for the atom to stay in the ground state thus decreases as $e^{-\Gamma_{t} t}$ with $\Gamma_{t}=\Gamma_{1}+\Gamma_{2}+\Gamma_{g}$. We then pick a random number $0 \leqslant r \leqslant 1$ and consider that the atom will leave the ground state after a time $t$ given by

$$
t=-\frac{\ln r}{\Gamma_{t}}
$$

We then compare the normalized probabilities $P_{i}=\Gamma_{i} / \Gamma_{t}$ with $i=(1,2, g)$ to a new random value $r^{\prime}$ in order to decide what kind of transition the atom will make. If the transition is the ionization, this atom is eliminated from the distribution. If it is a transition to an intermediate state, we compute its new velocity: $v(t)=v(0) \pm \Delta$, taking into account the velocity shift due to the absorption of a photon in mode 1 or 2 .

An analogous procedure is applied to the excited atoms in order to decide when they will decay by spontaneous emission to the ground state emitting a photon in a random direction and to correct their velocities. After a few thousands of fluorescence cycles, we approach the stationary condition.

We show in Fig. 2 the dependence of the stationary temperature on the ionization strength parameter $g$. The individual points correspond to the results of the Monte Carlo simulation and the solid line is the plot of Eq. (3.21). The results are seen to be in very good agreement, even for high values of the ionization rate.

Figure 3 shows the dependence of the stationary temperature on the detuning, for negative detunings. For positive

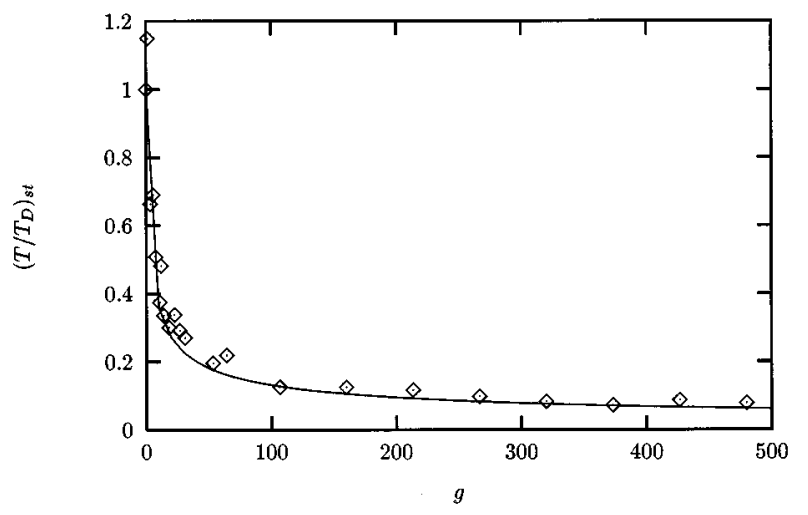

FIG. 2. Dependence of the stationary temperature on the ionization rate. These data correspond to $\delta=-\Gamma / 2$ and $\Omega=\Gamma$. The full line is a plot of Eq. (3.21), whereas the individual points correspond to the Monte Carlo simulation.

detunings the Doppler process leads to a nonstationary heating of the system, but the Monte Carlo simulations have shown that the selective ionization is still able to cool the system below the Doppler limit.

The Monte Carlo results have an uncertainty of about $\pm 10 \%$ essentially due to the relatively small number of atoms (a few hundreds) that can be dealt with in reasonable computing times. This is the cause of the dispersion of the points seen in Fig. 3.

As mentioned above, the main limitation of the IAOM is the decrease of the number of ground-state atoms as the temperature decreases. In Fig. 4 we plotted the number of remaining atoms after a time $\tau_{0}=2 \lambda^{-1}$ as a function of the temperature. As we have seen, the time constant for the temperature relaxation is $(2 \lambda)^{-1}$ [see Eq. (3.22)], and $T\left(\tau_{0}\right) / T_{s t} \approx 1.04$. A diminution of a factor of 50 in the temperature (which is the typical rate between the Doppler and the photon-recoil temperature) can thus be obtained at the price of a reduction of a factor of 20 in the number of atoms. Concluding this section, we can say that there is a good agreement between the theory introduced above and the Monte Carlo simulations, describing the IAOM below the Doppler limit temperature.

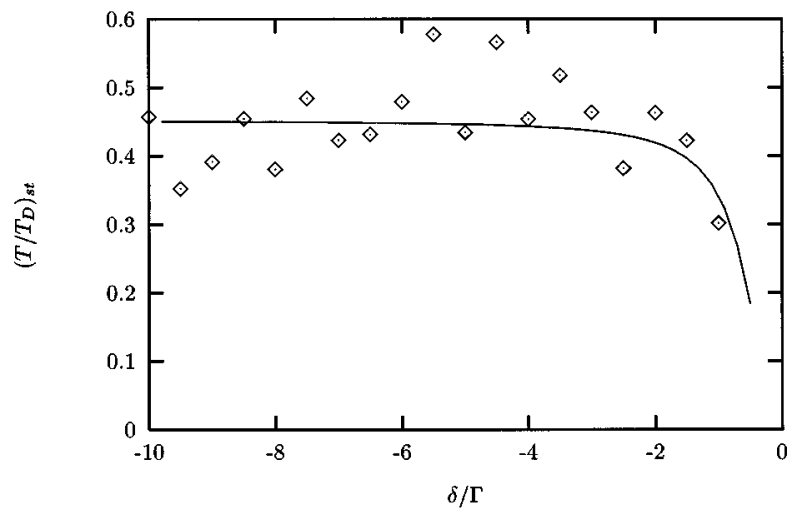

FIG. 3. Dependence of the stationary temperature on the detuning. $\Omega=\Gamma, g=100$. The full line is obtained from Eqs. (3.21), (3.19), (3.14); the individual points correspond to the Monte Carlo simulation. 


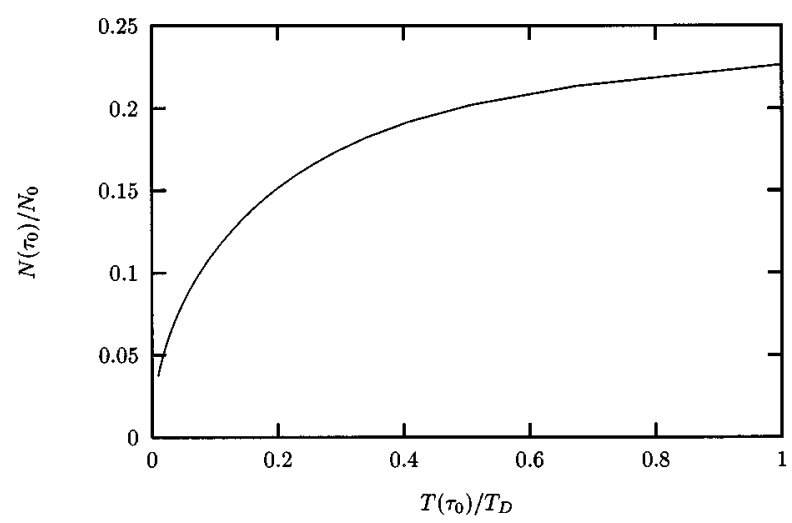

FIG. 4. Number of remaining atoms at time $\tau_{0}=2 \lambda^{-1}$ as a function of the temperature.

\section{IONIZATION ASSISTED OPTICAL MOLASSES WITH A SOURCE OF ATOMS}

As we have seen in Sec. III, the IAOM leads to a stationary temperature below the Doppler equilibrium temperature that is roughly proportional to $g^{-1 / 2}$. On the other hand, it has been seen that the number of cold atoms tends asymptotically to zero, which constitutes the main limitation of the proposed method. In the present section we shall study the case where "new" ground-state atoms are continuously injected into the system in order to replace the atoms lost by ionization. We suppose that these new atoms have been previously cooled by a standard Doppler molasses to the Doppler temperature.

Within this hypothesis, the kinetic equation for the IAOM can be easily generalized by adding a source term

$$
\frac{\partial n}{\partial \tau}=\frac{\partial(V n)}{\partial V}+\frac{\partial^{2} n}{\partial V^{2}}-g V^{2} n+\frac{s}{\sqrt{2 \pi}} e^{-V^{2} / 2}
$$

where $s$ is a scaled source intensity (in usual units $s$ is the atom flux per velocity damping time $\tau_{v}=M / \alpha$ ).

We did not find an analytical solution for this equation, but a certain number of relevant information can be obtained by deriving evolution equations for the temperature and the total number of atoms. The total number of ground-state atoms at time $\tau$ is given by

$$
N(\tau)=\int d V n(V, \tau)
$$

and thus

$$
\dot{N}=\int d V \frac{\partial n(V, \tau)}{\partial \tau}
$$

whereas the mean quadratic velocity (that is equal to $T / T_{D}$ in our normalized units) is

$$
x(\tau)=\left\langle V^{2}\right\rangle=\frac{1}{N} \int d V V^{2} n(V, \tau)
$$

leading to

$$
\dot{N} x+N \dot{x}=\int d V V^{2} \frac{\partial n(V, \tau)}{\partial \tau} .
$$

One can then substitute the kinetic equation (5.1) into these expressions. The terms containing partial derivatives with respect to the velocity can be integrated by parts, and the following equations are obtained for the time evolution of $x$ and $N$ :

$$
\dot{N}=-g x N+s
$$

and

$$
N \dot{x}=-g\left(\left\langle V^{4}\right\rangle-x^{2}\right) N-2 N x+2 N+s(1-x) .
$$

This equation shows that, due to the presence of the ionization term, the equation for $\left\langle V^{2}\right\rangle$ involves $\left\langle V^{4}\right\rangle$. Let us thus define the quantity $z$ possibly depending on $\tau$ and $g$

$$
z=\frac{\left\langle V^{4}\right\rangle}{\left\langle V^{2}\right\rangle^{2}}
$$

We now search for the stationary solutions of Eqs. (5.6) and (5.7). The quantity $z(\tau)$, being the ratio of two mean values, should also approach a stationary value $z_{s t}$ if the velocity distribution does so. We thus keep $z$ as a free parameter inside the resulting equations. We find, for $g>2$,

$$
x_{s t}=\frac{g-2}{2 g z_{s t}}\left(1+\left[1+\frac{8 g z_{s t}}{(g-2)^{2}}\right]^{1 / 2}\right)
$$

and

$$
N_{s t}=\frac{2 s z_{s t}}{g-2} \frac{1}{1+\left[1+\frac{8 g z_{s t}}{(g-2)^{2}}\right]^{1 / 2}},
$$

where we note that $x_{s t}$ does not depend on $s$. This result is not surprising: the original kinetic equation (5.1) can be divided by $s$, which corresponds to a renormalization of the velocity distribution $n(V, \tau) \rightarrow n(V, \tau) / s$ that does not affect the mean values. This means that we can have as many atoms as we want in the stationary state (taking a large enough source term) without changing the limit temperature. On the other hand, the time necessary to attain the stationary state will be correspondingly increased.

Asymptotically $(g \rightarrow \infty), x_{s t} \rightarrow 1 / z_{s t}$. Thus, the asymptotic value of the temperature depends on $g$ only implicitly, which means that this dependence is due to the fact that the stationary velocity distribution changes shape with $g$. For a Gaussian-shaped distribution $z=3$ regardless of the value of the velocity dispersion.

The important point now is that the stationary solution for the total number of ground-state atoms does not vanish as was the case in the absence of a source of new atoms: $N_{s t} \rightarrow s /\left(g z_{s t}\right)$. Our main problem is thus to determine the dependence of $z_{s t}$ on $g$.

In principle, there is no general relation between $\left\langle V^{2}\right\rangle$ and $\left\langle V^{4}\right\rangle$ for an arbitrary distribution. We thus used the Monte Carlo method discussed in Sec. IV, in which we have included the source term, to determine numerically the relation between $z_{s t}$ and $g$. It turns out (see Fig. 5) that the numerical 


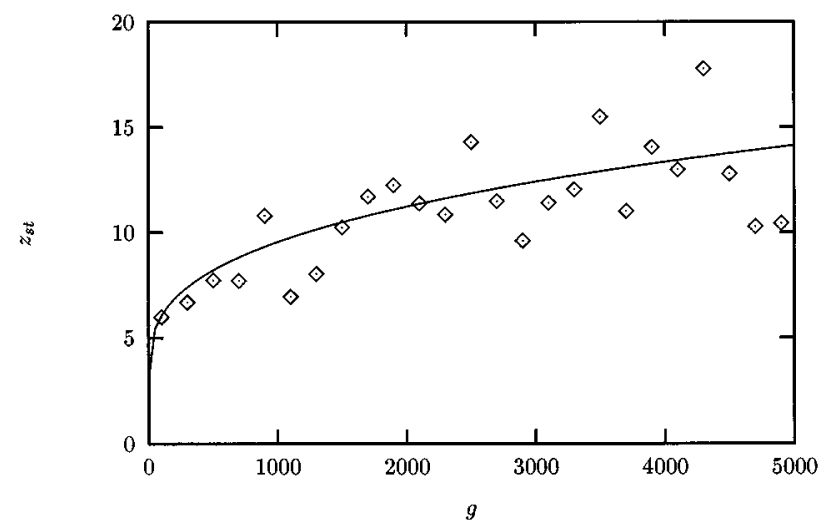

FIG. 5. Dependence of $z_{s t}=\left\langle V^{4}\right\rangle_{s t} /\left\langle V^{2}\right\rangle_{s t}^{2}$ on $g$. The fitting curve is $z_{s t}=3+(2 / 3) g^{1 / 3}$. The increasing fluctuations of the numerical results for high $g$ are due to the decrease in the number of atoms at the stationary state (cf. text). These data correspond to $\delta=-\Gamma / 2, \Omega=\Gamma / 2$, and $g \geqslant 100$. The source term corresponds to $10^{8}$ atoms/s. The full curve corresponds to Eq. (5.11) and the points to the Monte Carlo results.

results are correctly fitted by the following relation (remember that $z=3$ corresponds to a Gaussian distribution):

$$
z_{s t}=3+\frac{2}{3} g^{1 / 3}
$$

As one sees in the figure, there is an increasing uncertainty on the results of the numerical simulation when the ionization rate increases. This is due to the decrease in the number of remaining ground-state atoms when the stationary state is attained, for high ionization rates. As we have mentioned before, these results are independent of the value of the source intensity $s$. This has been confirmed by the numerical simulation.

Once we have determined the dependence of $z_{s t}$ on $g$, we can insert this expression back in Eqs. (5.9) and (5.10). We thus see that the temperature goes asymptotically $(g \rightarrow \infty)$ as $g^{-1 / 3}$. We plotted in Fig. 6 the dependence of the stationary temperature on the ionization rate. The curve fits rather well the results of the Monte Carlo simulation. Figure 7 shows the

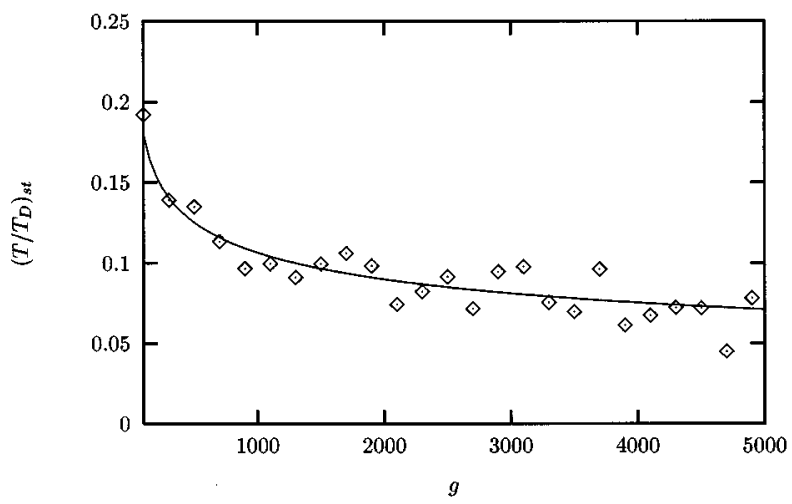

FIG. 6. Comparison between the stationary-state temperature obtained from Eqs. (5.9) and (5.11) and the numerical simulation. $\delta=-\Gamma / 2, \Omega=\Gamma / 2, g \geqslant 100$. The source intensity is $10^{8}$ atoms/s.

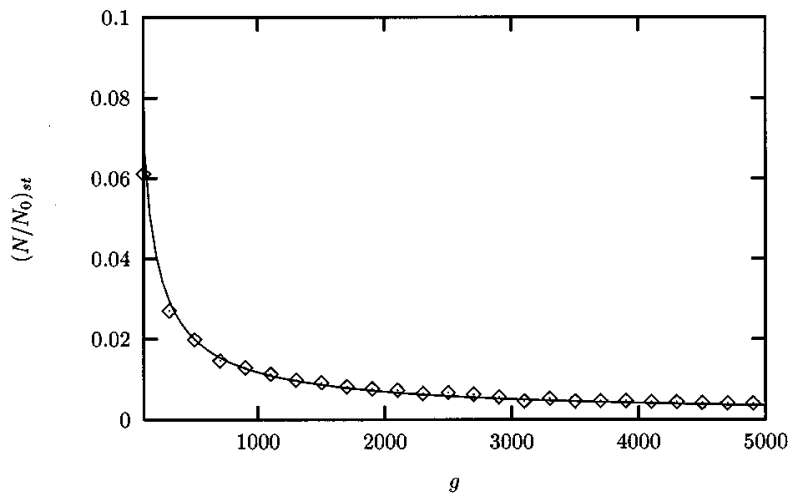

FIG. 7. Comparison between the stationary-state number of atoms obtained from Eqs. (5.10) and (5.11) and the numerical simulation. $\delta=-\Gamma / 2, \Omega=\Gamma / 2, g \geqslant 100$. The source intensity is $10^{8}$ atoms/s.

same for the number of atoms relative to the initial value $N_{0}$. Once again there is a good agreement with the simulation.

A good parameter characterizing the performance of the velocity selection is the phase-space density defined as the ratio of the number of atoms per unit velocity interval (we are supposing that there is no trapping effect, so that the spatial coordinates are not affected by the velocity selection process), that can be approximated by

$$
\rho \approx \frac{N}{\Delta V} \approx \frac{N}{\sqrt{x}} .
$$

We can thus calculate the time derivative of the phase-space density

$$
\dot{\rho}=\frac{N}{\sqrt{x}}\left(\frac{s}{N}-g x-\frac{\dot{x}}{2 x}\right),
$$

where we used Eq. (5.6) in order to eliminate the time derivative of the number of atoms. One sees that the phasespace density will increase as the temperature diminishes provided

$$
\frac{s}{N}>g x+\frac{1}{2} \frac{\partial \ln x}{\partial \tau} .
$$

As the temperature tends to its stationary value, the second term in the right-hand side tends to zero, and the lowering of the temperature tends to decrease the first term, so that it becomes easier and easier to fulfill the density-increasing condition. For example, let us take $g=10^{4}$. Then $x_{s t} \approx 0.02$ which is of the order of the photon-recoil temperature; the density increasing condition is fulfilled if $s \approx 200$, which, in usual units, means that in order to have $10^{8}$ atoms in the stationary state (the usual value obtained with other methods), one must have a source term of about $2 \times 10^{13}$ atoms per second (we take $\tau_{v}=1 \mathrm{~ms}$ ), which is a realistic flux in a laser-cooled atomic beam experiment.

Concluding this section, we can say that the "source" scheme for the IAOM shows interesting characteristics: although the stationary value of the temperature decreases with 
the power $1 / 3$ of the ionization rate instead of $1 / 2$ as was the case in the absence of the source, the number of cold atoms does not vanish, but tends to a stationary value that decreases with $g^{-2 / 3}$. We have also shown that the condition for an increasing of the phase-space density during the velocity selection process can be fulfilled in realistic conditions.

\section{TWO-DIMENSIONAL SCHEME FOR GROUND-STATE DEGENERATE ATOMS}

The method proposed in this paper is in principle able to cool atoms to temperatures comparable or even smaller than the "photon-recoil energy limit" - naturally at the price of either an important decrease in the number of atoms or of coupling the velocity selection scheme to a relatively bright source of Doppler-temperature atoms. These limitations can be overcome by starting with the atoms close to the photonrecoil energy in a molasses presenting the so-called "Sisyphus effect"' $[1,16,17]$. Nevertheless, the analysis presented here does not apply to such a molasses, because the Sisyphus effect exists only for ground-state degenerate atoms. Moreover, the Fokker-Planck type approach leads, in the case of a degenerate ground state, to coupled equations [16] that are difficult to solve, although the inclusion of the ionization effect turns out to be straightforward. Also, the numerical analysis of such problems involves the using of quantum Monte Carlo techniques [17]. For these reasons, we shall restrict ourselves in the present section to proposing schemes for combining the present velocity selection method with Sisyphus-type mechanisms for a ground-state degenerate atom. We shall study the destructive interference conditions, without giving a quantitative analysis of the performances of such system; a thorough semiclassical analysis will be published elsewhere [18].

An interesting ground-state degenerate system is a $J_{g}=1$ atom with a $J_{e}=1$ intermediate state $(J$ being the angular momentum), which is the case of metastable ${ }^{4} \mathrm{He} *$ or ${ }^{87} \mathrm{Rb}$. We show in Fig. 8 the allowed transitions and the respective Clebsch-Gordan coefficients for such a system. This scheme presents a two-photon coupling to a discrete upper level, itself coupled to the continuum, instead of a direct coupling to the continuum. This has many advantages [8]: first, it allows one to get rid of the possible paths leading from the ground state to the continuum via the absorption of two photons in the same mode, which are not subjected to interference effects, simply by choosing a $J_{s}=0$ upper level, as shown in Fig. 8. Moreover, it allows us to turn the ionization on and off by switching the ionizing laser; one can thus wait for the system to attain the Sisyphus equilibrium temperature before switching on the ionization. It is easy to understand (and this can be rigorously demonstrated [18]) that if ionizing coupling between the upper level $|s\rangle$ and the continuum is high enough (i.e., if the ionization rate is large compared to the natural width of the upper level), once the system arrives on the upper level it will have a great probability of being ionized, and the dynamics of this system will be much like that of the system shown if Fig. 1.

We propose here a two-dimensional scheme allowing a combination with Sisyphus mechanisms. It is worth mentioning that Sisyphus-type mechanisms in one dimension for a $J=1 \rightarrow J=1$ transition present a certain number of "patho-
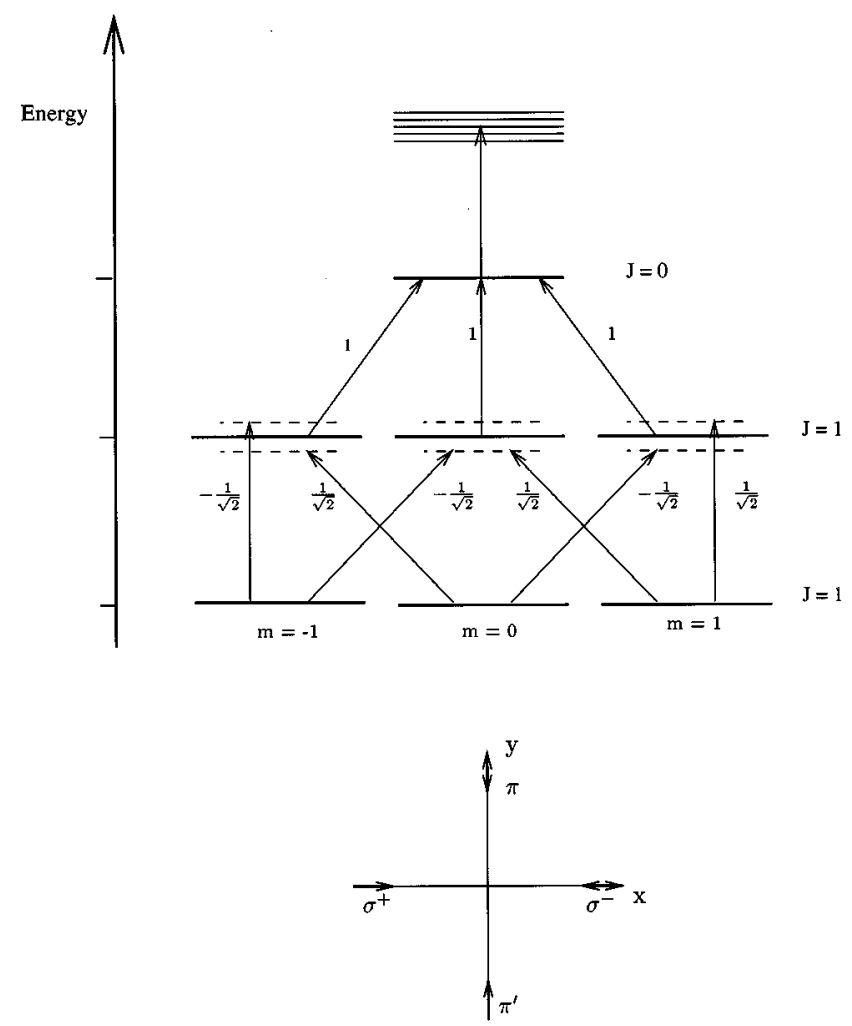

FIG. 8. Two-dimensional arrangement for the combination of velocity selection by ionization with Sisyphus-type mechanisms in a $J_{g}=1 \rightarrow J_{e}=1 \rightarrow J_{s}=0$ level structure.

logical cases," in which there is no Sisyphus effect, that should be avoided. In general, it is easier to obtain Sisyphus configurations in two or three dimensions. For more details, see Refs. $[16,19]$. For simplicity, we shall call, in what follows, "Sisyphus mechanisms" both the proper Sisyphus mechanism obtained with counterpropagating, orthogonally polarized, laser waves, based on light-shift effects, and the alignment, motion-sensitive, mechanism obtained in $\sigma^{+}-\sigma^{-}$schemes [1].

Many different schemes can be conceived. The important points are: (1) One must have polarization-alignment gradients in order to obtain the Sisyphus effect; (2) one must combine circularly and linearly polarized beams in order that the ionization be effective for all ground-state sublevels; and (3) one must find destructive interference conditions in the two-photon transitions able to select the desired velocity class. As an illustration, consider, for example, a scheme where one uses $\sigma^{+}-\sigma^{-}$counterpropagating waves along the $x$ axis and orthogonally polarized counterpropagating waves along the $y$ axis (Fig. 8). The $m_{g}=0$ atoms interact with the $\sigma^{+}-\sigma^{-}$waves and velocity selection along the $x$ axis is performed as before. Moreover, the selected $m_{g}=0$ atoms can be optically pumped in the other sublevels. The atoms in the $m_{g}= \pm 1$ sublevels can also make two-photon transitions by absorption of one photon from one of the $\sigma$-polarized, $x$-propagating waves and another photon from one of the $\pi$-polarized, $y$-propagating waves. In order to study the interference conditions, consider, for definiteness, the case of the $m_{g}=1$ level. We show in Fig. 9 the Feynman diagrams for all possible two-photon processes starting from 

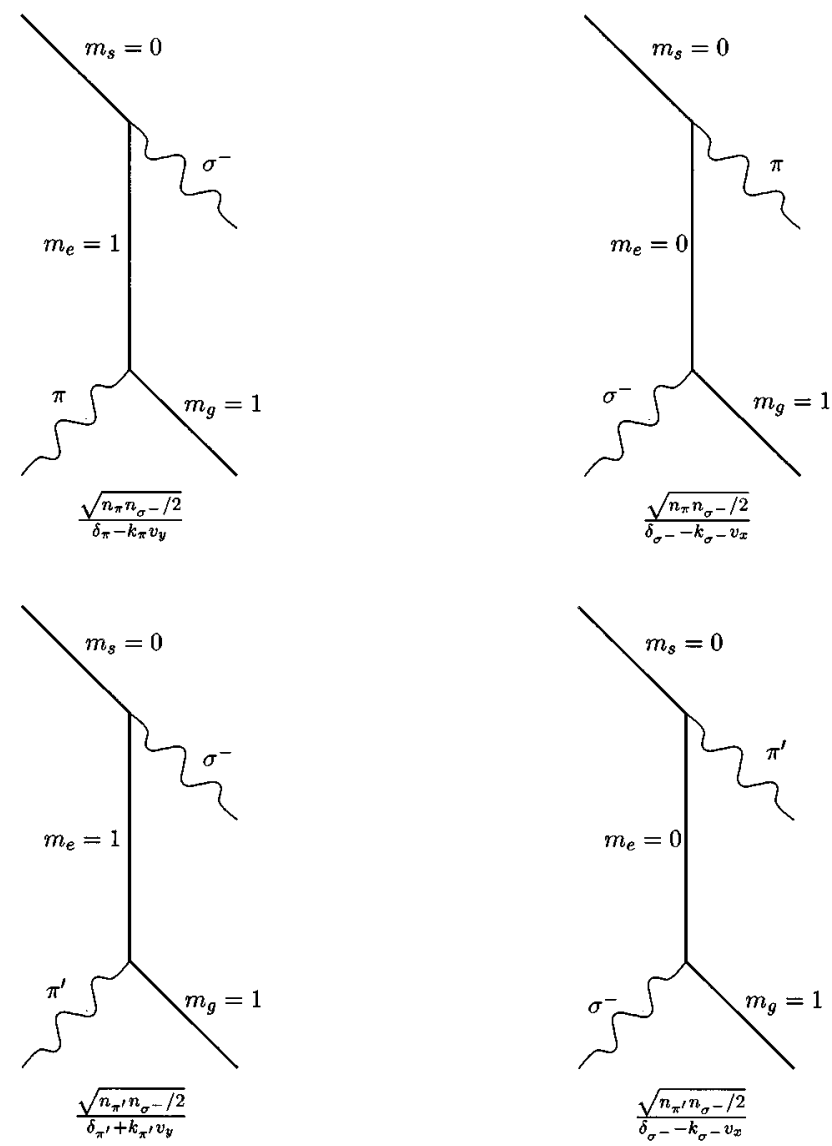

FIG. 9. Feynman diagrams corresponding to the two photon processes starting from the sublevel $m_{g}=1$ of Fig. 8, with the corresponding transition amplitudes.

this level, together with the related probability amplitudes. One sees that there are four possible paths, leading to two different final states: the atom can absorb a $\sigma^{-}$and a $\pi$ photon, and the temporal order of these absorptions can be exchanged. This corresponds to the two top diagrams in Fig. 9, that lead to the same global (atom+field) final state. The two bottom diagrams are analogous, except that the linearly polarized photon comes from the $\pi^{\prime}$ wave, and leads to a final state distinct of the preceding one. The ionization rate for this state thus consists of four terms interfering two by two

$$
\begin{aligned}
\Gamma_{g}\left(m_{g}=1\right)= & \bar{\Gamma} \omega^{2}\left[\frac{n_{\sigma^{-}} n_{\pi}}{2}\left|\frac{1}{\delta_{\pi}-k_{\pi} v_{y}}+\frac{1}{\delta_{\sigma^{-}}-k_{\sigma^{-}} v_{x}}\right|^{2}\right. \\
& \left.+\frac{n_{\sigma^{-}} n_{\pi^{\prime}}}{2}\left|\frac{1}{\delta_{\pi^{\prime}}+k_{\pi^{\prime}} v_{y}}+\frac{1}{\delta_{\sigma^{-}}-k_{\sigma^{-}} v_{x}}\right|^{2}\right] .
\end{aligned}
$$

One then sees that the ionization rate vanishes if the atom satisfies two destructive interference conditions.

$$
\begin{gathered}
\delta_{\pi}-k_{\pi} v_{y}=-\delta_{\sigma^{-}}+k_{\sigma^{-}} v_{x} \\
\delta_{\pi^{\prime}}+k_{\pi^{\prime}} v_{y}=-\delta_{\sigma^{-}}+k_{\sigma^{-}} v_{x}
\end{gathered}
$$

Furthermore, these conditions, that mix the $v_{x}$ and $v_{y}$ components of the atomic velocity, are simultaneously satisfied for zero velocity atoms (and only zero velocity atoms) if the detunings are such that $\delta_{\pi^{\prime}}=\delta_{\pi}=-\delta_{\sigma^{-}}$. It can be easily shown that the same condition is valid for the $m_{g}=-1$ level. The $\sigma$-polarized waves should thus have opposite detunings with respect to the $\pi$-polarized waves. It can be thus necessary to readjust the detunings at the same time as the ionizing laser is turned on, once the system attains the Sisyphus equilibrium temperature. Note, moreover, that this scheme can be extended to three dimensions simply by adding a third couple of $\sigma^{+}-\sigma^{-}$or orthogonally polarized waves along the $z$ axis.

Before concluding this section, let us note that other similar two- or three-dimensional schemes can be conceived; for example, one can use counterpropagating $\sigma^{ \pm}-\pi$ waves along each axis. This system also satisfies the three conditions mentioned above: it leads to polarization gradients, it allows optical pumping among the various ground-state sublevels, and one can easily work out the destructive interference conditions for selecting $v=0$ atoms.

\section{CONCLUSION}

The aim of this paper was to study a process of velocity class selection using quantum interference in the two-photon ionization of an atomic ground state. In particular, we studied the effect of the velocity redistribution due to spontaneous emission from the intermediate atomic levels in a "ionization-assisted" optical molasses. The main result we obtained is that the temperature tends to a stationary value, in contrast with the case where spontaneous emission is negligible. This stationary value is asymptotically proportional to the inverse square root of the ionization strength; it is thus in principle possible to have arbitrarily low temperature if the ionization strength is high enough. We saw, however, that due to the irreversible nature of the ionization process, low temperatures correspond to small numbers of remaining atoms, and this is the major limitation of this method. We have also shown that this limitation can be eliminated by using a source regime in which "new" atoms at the Doppler temperature are continuously injected into the system. This leads to a stationary state at a higher temperature but with a constant number of ground-state atoms. Moreover, we showed that one can find realistic conditions in which the cooling by velocity selection is accompanied by an increasing of the phase-space density.

We also proposed a generalization of the velocity selection by quantum interference for ground-state degenerate atoms, and we are presently working on a semiclassical analysis of such a system. It is, however, worthwhile to note that the photon-recoil energy is the limit of validity of the semiclassical approaches, and a full quantum analysis would be desirable.

The main difficulty with the present method is naturally the depopulation of the ground state by the ionization, that imposes the use of a high initial atomic density or the coupling of the system to a relatively bright source of Dopplertemperature atoms. However, let us mention that the other methods that have been experimentally demonstrated to produce sub-recoil temperatures are also plagued by consider- 
able losses in the number of cooled atoms. In VSCPT, for example, it can be shown theoretically that the probability of trapping an atom increases linearly with the interaction time [20], so that eventually all atoms are trapped. However, this method is based on the creation of a ground-state quantum coherence that is very sensitive to external perturbations, so that the efficiency of the trapping is limited by, e.g., collisions with untrapped atoms. With the Raman method, the efficiency of the cooling is limited, among other factors, by the quality of the shaping of the laser pulses necessary to avoid off-resonant excitations. Subrecoil temperatures have been obtained in this way together with an increasing of a factor of about 20 in the phase-space density [21]. This is, to our knowledge, the best performance yet obtained. Finally, in evaporative cooling experiments, that are based on selective ejection of hot atoms by elastic collisions, there is also an important decreasing in the number of trapped atoms. Hulet and co-workers have observed a number of trapped atoms proportional to $T^{-3 / 2}$ [6]. Such performances are arguably attainable with the source version of the present method.
On the other hand, the method proposed here is very robust: it is insensitive to collisions, it does not depend on highly stabilized lasers and the ionizing laser powers, although high, are available from commercial lasers. Moreover, the crucial factor $g$ (the ionization strength) is proportional to $(\Omega / \delta)^{2}$ [Eq. (3.14)]. This means that it can be very high even for modest laser intensities (that are proportional to $\Omega^{2}$ ) by choosing a small enough detuning (quasiresonant process). In this case, the maximum value of $g$ is limited only by the natural lifetime of the intermediate level.

\section{ACKNOWLEDGMENTS}

The authors gratefully acknowledge useful discussions with Y. Castin. Laboratoire de Spectroscopie Hertzienne is Unité de Recherche associé au CNRS. Centre d'Études et de Recherches Lasers et Applications is supported by the Ministère de la Recherche, Région Nord-Pas de Calais, and Fonds Europeen de Développement Économique des Régions.
[1] P. Lett, R. Watts, C. Westbrook, W. D. Phillips, P. Gould, and H. Metcalf, Phys. Rev. Lett. 61, 168 (1988); Y. Shevy, D. S. Weiss, P. J. Ungar, and S. Chu, ibid. 62, 1118 (1989); J. Dalibard and C. Cohen-Tannoudji, J. Opt. Soc. Am. B 6, 2023 (1989); P. J. Ungar, D. S. Weiss, E. Riis, and S. Chu, ibid. 6, 2058 (1989).

[2] A. Aspect, E. Arimondo, R. Kaiser, N. Vansteenkiste, and C. Cohen-Tannoudji, Phys. Rev. Lett. 61, 826 (1988).

[3] M. Kasevich and S. Chu, Phys. Rev. Lett. 69, 1741 (1992).

[4] H. F. Hess, Phys. Rev. B 34, 3476 (1986); K. B. Davis, M. O. Mewes, M. A. Joffe, M. R. Andrews, and W. Ketterle, Phys. Rev. Lett. 74, 5202 (1995).

[5] M. H. Anderson, J. R. Ensher, M. R. Matthews, C. E. Wieman, and E. A. Cornell, Science 269, 198 (1995).

[6] C. C. Bradley, C. A. Sackett, J. J. Tollet, and R. G. Hulet, Phys. Rev. Lett. 75, 1687 (1995).

[7] K. B. Davis, M. O. Mewes, M. R. Andrews, N. J. Vandruten, D. M. Kuru, and W. Ketterle, Phys. Rev. Lett. 75, 3969 (1995).

[8] J. C. Garreau, Phys. Rev. A 53, 486 (1996).

[9] T. W. Hänsch and A. L. Schawlow, Opt. Commun. 22, 373 (1975).

[10] R. J. Cook, Phys. Rev. A 22, 1078 (1980).

[11] C. Cohen-Tannoudji, in Fundamental Systems in Quantum Optics, Les Houches School 1990, edited by J. Dalibard, J. M. Raimond, and J. Zinn-Justin (North-Holland, Amsterdam, 1992).
[12] J. Dalibard and C. Cohen-Tannoudji, J. Phys. B 18, 1661 (1985).

[13] F. Reif, Fundamentals of Statistical and Thermal Physics (MacGraw-Hill, Kogakusha, Tokyo, 1965).

[14] Equation (3.16) is valid only if $\alpha>0$, i.e., if $\delta<0$ (which corresponds to the case of Doppler cooling; if $\delta>0$ the laser heats the atoms). If $\delta>0$, the equation takes the form

$$
\frac{\partial n}{\partial \tau}=-\frac{\partial(V n)}{\partial V}+\frac{\partial^{2} n}{\partial V^{2}}-g V^{2} n
$$

with the same scaling conventions taken with $\alpha \rightarrow|\alpha|$.

[15] The Monte Carlo method was first introduced by M. N. Rosenbluth and A. W. Rosenbluth, J. Chem. Phys. 31, 369 (1959). For an application to the magneto-optical trap, see Delphine Grison, thèse de doctorat, Paris, 1992 (unpublished).

[16] Y. Castin, K. Berg-Sorensen, J. Dalibard, and K. Mфlmer, Phys. Rev. A 50, 5092 (1994).

[17] Y. Castin and K. Mølmer, Phys. Rev. Lett. 74, 3772 (1995).

[18] V. Zehnlé and J. C. Garreau (unpublished).

[19] P. Marte, R. Dum, R. Taïeb, P. Zoller, M. S. Shahriar, and M. Prentiss, Phys. Rev. A 49, 4826 (1994); M. Weidemüler, T. Esslinger, M. A. Ol'shanii, A. Hemmerich, and T. W. Hänsch, Europhys. Lett. 27, 109 (1994).

[20] F. Bardou, J. P. Bouchaud, O. Emile, A. Aspect, and C. Cohen-Tannoudji, Phys. Rev. Lett. 72, 203 (1994).

[21] N. Davidson, H. J. Lee, M. Kasevich, and S. Chu, Phys. Rev. Lett. 72, 3158 (1994). 